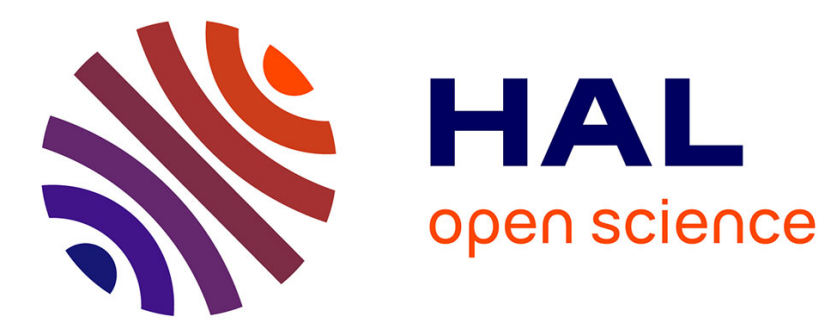

\title{
Effective elastic properties of auxetic microstructures : anisotropy and structural applications
}

\author{
Justin Dirrenberger, Samuel Forest, Dominique Jeulin
}

\section{To cite this version:}

Justin Dirrenberger, Samuel Forest, Dominique Jeulin. Effective elastic properties of auxetic microstructures : anisotropy and structural applications. International Journal of Mechanics and Materials in Design, 2013, 9 (1), pp.21-33. 10.1007/s10999-012-9192-8 . hal-00790180

HAL Id: hal-00790180

https://hal-mines-paristech.archives-ouvertes.fr/hal-00790180

Submitted on 23 Dec 2019

HAL is a multi-disciplinary open access archive for the deposit and dissemination of scientific research documents, whether they are published or not. The documents may come from teaching and research institutions in France or abroad, or from public or private research centers.
L'archive ouverte pluridisciplinaire HAL, est destinée au dépôt et à la diffusion de documents scientifiques de niveau recherche, publiés ou non, émanant des établissements d'enseignement et de recherche français ou étrangers, des laboratoires publics ou privés. 


\title{
Effective elastic properties of auxetic microstructures: anisotropy and structural applications
}

\author{
Justin Dirrenberger $\cdot$ Samuel Forest $\cdot$ \\ Dominique Jeulin
}

Received: 29 November 2011 / Accepted: 24 April 2012/Published online: 26 May 2012

(C) Springer Science+Business Media, B.V. 2012

\begin{abstract}
Materials presenting a negative Poisson's ratio (auxetics) have drawn attention for the past two decades, especially in the field of lightweight composite structures and cellular media. Studies have shown that auxeticity may result in higher shear modulus, indentation toughness and acoustic damping. In this work, three auxetic periodic microstructures based on 2D geometries are considered for being used as sandwich-core materials. Elastic moduli are computed for each microstructure by using finite elements combined with periodic homogenization technique. Anisotropy of elastic properties is investigated in and out-of-plane. Comparison is made between auxetics and the classical honeycomb cell. A new 3D auxetic lattice is proposed for volumic applications. Cylindrical and spherical elastic indentation tests are simulated in order to conclude on the applicability of such materials to structures. Proof is made that under certain conditions, auxetics can be competitive with honeycomb cells in terms of indentation strength. Their relatively soft response in
\end{abstract}

J. Dirrenberger $(\bowtie) \cdot$ S. Forest $\cdot$ D. Jeulin

Centre des Matériaux, MINES-ParisTech, CNRS UMR

7633, BP 87, 91003 Evry Cedex, France

e-mail: justin.dirrenberger@mines-paristech.fr

D. Jeulin

Centre de Morphologie Mathématique, MINES-

ParisTech, 35, rue Saint-Honoré, 77305 Fontainebleau,

France tension can be compensated, in some situations, by high shear moduli.

Keywords Homogenization · Finite element method - Auxetics · Anisotropy · Negative Poisson's ratio - Indentation · Architectured materials · Elasticity

\section{Introduction}

In the case of isotropic elasticity, mechanical behavior is described by any couple of parameters among these: Young's modulus $E$, Poisson's ratio $v$, bulk modulus $K$ and Lamé's coefficients $\lambda$ and $\mu$ (also referred to as $G$, shear modulus). When a material is loaded uniaxially in tension, Poisson's ratio is defined as the ratio of the contraction in the transverse direction to the extension in the longitudinal direction. Thermodynamically, $v$ lies between -1 , for unshearable materials, and 0.5 for incompressible or rubber-like materials. Most materials naturally present a positive Poisson's ratio, although negative Poisson's ratio materials, or auxetics (Evans et al. 1991a), have been engineered since the mid-1980s (Almgren 1985; Lakes 1987; Caddock and Evans 1989; Lakes 1991; Milton 1992; Prall and Lakes 1997; Gaspar et al. 2005; Alderson et al. 2010; Dirrenberger et al. 2011). Such materials have been expected to present enhanced mechanical properties such as shear modulus and fracture toughness (Choi 1996), indentation resistance (Lakes 1993; Alderson et al. 1994, 2000) but also 
acoustic damping (Lipsett and Beltzer 1988; Chen and Lakes 1996). The use of auxetics as building blocks for wave-guiding metamaterials has also been investigated by Spadoni et al. (2009). Besides, $v<0$ allows synclastic curvature of plates (Evans 1991b), thus enabling the manufacture of doubly-curved (domeshaped) sandwich panels without core buckling. Moreover auxetic foams seem to provide better resistance to crash than conventional cellular materials (Scarpa et al. 2002). Auxetics could thus be used in both functional and structural applications, we will only consider the latter in this work.

However, most auxetic materials built-up from periodic microstructures display strongly anisotropic properties so that auxeticity is observed only with respect to specific axes. The elastic anisotropy of such materials may not have been sufficiently analysed in the literature, this is the purpose of the present work.

This paper deals with the numerical determination of the effective elastic tensor components of three 2D microstructures, the investigation of their in-plane and out-of-plane anisotropy and the comparison with a honeycomb lattice. First, preliminary considerations regarding the homogenization framework are made in Sect. 2. Geometries of the auxetic microstructures and the classical honeycomb cell are presented in Sect. 3. Volumic finite elements (FE) coupled with periodic homogenization technique are then used in Sect. 4 to compute elastic moduli, characterize anisotropy and compare the different microstructures considered. A new tridimensional auxetic periodic microstructure is proposed and studied in Sect. 5. Structural applicability of auxetics is validated in Sect. 6 with the simulation of cylindrical and spherical elastic indentation tests. Finally the use of such materials in terms of design and engineering applications is put into perspective in Sect. 7.

\section{Numerical homogenization}

In this work, numerical homogenization consists in determining effective mechanical properties over a unit-cell (defined by its periodicity vectors $v_{i}$ ) with periodic boundary conditions (PBC) using FE as in references (Kanit et al. 2003; Madi et al. 2005; Jean and Engelmayr 2010). Such an approach is quite popular among the mechanics of composites community, while scarce in auxetics research. Homogenization requires separation between micro and macro scales. In the case of periodic homogenization, the computed effective properties correspond to those of an infinite continuum made of periodic tiles.

The macroscopic stress and strain tensors $\underset{\sim}{\Sigma}$ and $\underset{\sim}{E}$ are defined by the spatial averages over the unit-cell of volume $V$ of local stress $\sigma$ and strain $\varepsilon$ fields:

$\underset{\sim}{\Sigma} \hat{=}\langle\underset{\sim}{\sigma}\rangle=\frac{1}{V} \int_{V} \underset{\sim}{\sigma} d V$

$\underset{\sim}{E} \hat{=}\langle\underset{\sim}{\varepsilon}\rangle=\frac{1}{V} \int_{V} \underset{\sim}{\varepsilon} d V$

PBC over the unit-cell give displacement field $\underline{u}$ such as:

$\underline{u}=\underset{\sim}{E} \cdot \underline{x}+\underline{v} \quad \forall \underline{x} \in V$

with $\underline{x}$, the material point location vector and $\underline{v}$, the periodic fluctuation. $\underline{v}$ takes the same value at two homologous points on opposite faces of $V$, whereas the traction vector $\underline{t}=\underset{\sim}{\sigma} \cdot \underline{n}$ takes opposite values, $\underline{n}$ being the normal vector.

By applying either macroscopic strain or stress, one can compute the effective fourth-rank tensors of elastic moduli $\underset{\approx}{C}$ and compliances $\underset{\approx}{S}$ defined such as:

$\underset{\sim}{\Sigma}=\underset{\approx}{C}: \underset{\sim}{E}$
$\underset{\sim}{E}=\underset{\approx}{S}: \underset{\sim}{\Sigma}$

Elastic moduli are written using Voigt's notation as follows:

$\left[\begin{array}{l}\Sigma_{11} \\ \Sigma_{22} \\ \Sigma_{33} \\ \Sigma_{23} \\ \Sigma_{31} \\ \Sigma_{12}\end{array}\right]=\left[\begin{array}{llllll}C_{11} & C_{12} & C_{13} & C_{14} & C_{15} & C_{16} \\ C_{12} & C_{22} & C_{23} & C_{24} & C_{25} & C_{26} \\ C_{13} & C_{23} & C_{33} & C_{34} & C_{35} & C_{36} \\ C_{14} & C_{24} & C_{34} & C_{44} & C_{45} & C_{46} \\ C_{15} & C_{25} & C_{35} & C_{45} & C_{55} & C_{56} \\ C_{16} & C_{26} & C_{36} & C_{46} & C_{56} & C_{66}\end{array}\right]\left[\begin{array}{c}E_{11} \\ E_{22} \\ E_{33} \\ 2 E_{23} \\ 2 E_{31} \\ 2 E_{12}\end{array}\right]$

\section{Microstructures considered}

\subsection{Hexachiral lattice}

This chiral microstructure was first proposed by Lakes in 1991, then studied in Prall and Lakes (1997), 
Alderson et al. (2010), Dirrenberger et al. (2011), Spadoni (2008). Based on the parameters defined in reference Alderson et al. (2010), cell geometry can be described in this way: the circular nodes have radius $\mathrm{r}$, the ligaments have length $\mathrm{L}$, and both have in common wall thickness $t$ (cf. Fig. 1a) as well as depth $\mathrm{d}$, which in our case is considered infinite due to periodicity conditions along direction 3 . Hence, three dimensionless parameters can be derived as shown in Eq. 7.

$\alpha=\mathrm{L} / \mathrm{r} \quad \beta=\mathrm{t} / \mathrm{r} \quad \gamma=\mathrm{d} / \mathrm{r}$

On Fig. $1 b, \alpha=5, \beta=0.25$ and $\gamma \rightarrow+\infty$. These parameters correspond to a volume fraction of $15 \%$. The sixfold symmetry provides transverse isotropy.

\subsection{Anti-tetrachiral lattice}

This microstructure was proposed and studied in (Alderson et al. 2010). Cell geometry can be described exactly as for the hexachiral lattice (cf. Fig. 2a). Here,

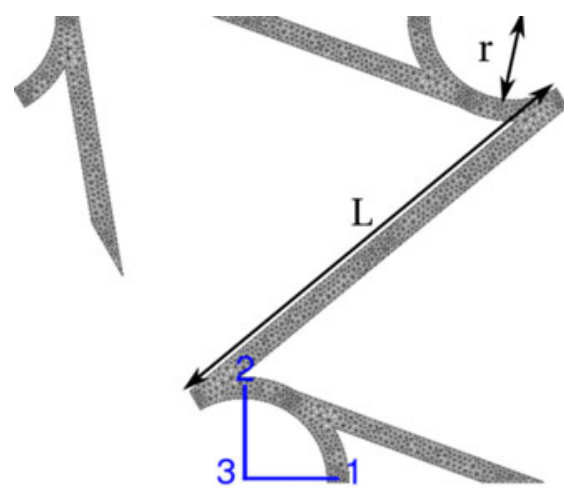

(a) Hexachiral unit-cell

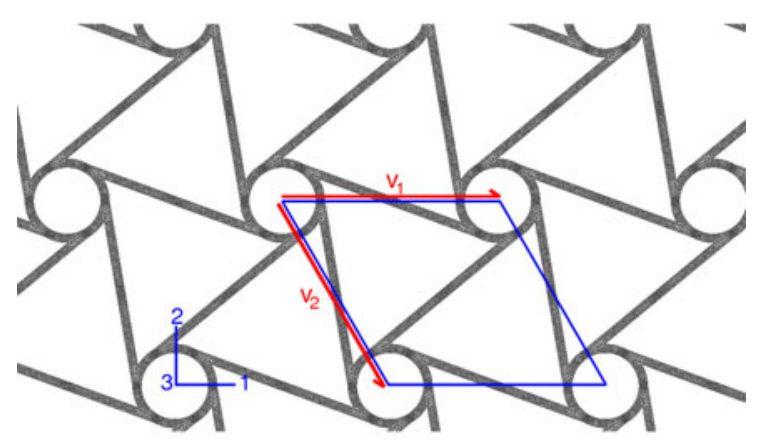

(b) Hexachiral lattice

Fig. 1 a Periodic cell with geometric parameters. b Hexachiral lattice with unit-cell (blue) and periodicity vectors $\underline{v_{1}}$ and $\underline{v_{2}}$ (red). (Color figure online) $\alpha=11, \beta=0.06$ and $\gamma \rightarrow+\infty$ (cf. Fig. 2b). Volume fraction is $15 \%$. The cell presents three orthogonal planes of symmetry, which gives rise to orthotropic elasticity.

\subsection{Rotachiral lattice}

This chiral microstructure, initially proposed in Dirrenberger et al. (2011), has been designed based on ideas from Prall and Lakes (1997) and Gaspar et al. (2005), the aim was to study the impact of ligaments geometry on auxeticity for chiral lattices. Cell geometry is similar to the hexachiral case, except for the straight ligaments that have been replaced by circular arcs with diameter D (cf. Fig. 3a). A new dimensionless parameter is defined:

$\delta=\mathrm{D} / \mathrm{r}$

As shown on Fig. $3 b, \delta=2.4, \beta=0.1$ and $\gamma \rightarrow+\infty$. Volume fraction is $15 \%$. The sixfold symmetry provides transverse isotropy.

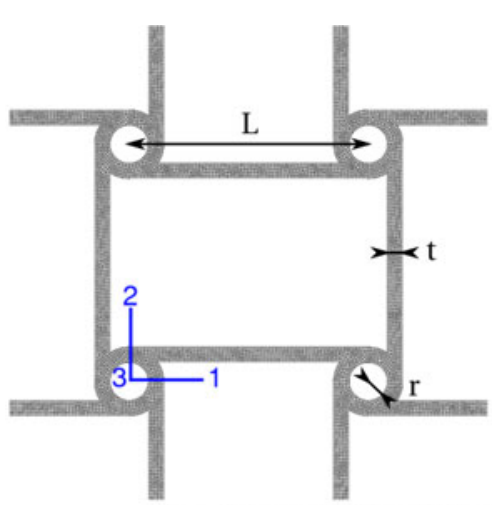

(a) Anti-tetrachiral unit-cell

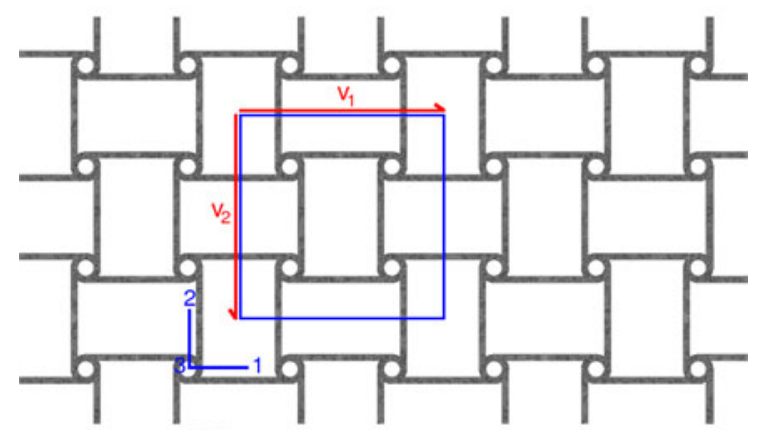

(b) Anti-tetrachiral lattice

Fig. 2 a Periodic cell with geometric parameters. b Antitetrachiral lattice with unit-cell (blue) and periodicity vectors $\underline{v_{1}}$ and $v_{2}$ (red). (Color figure online) 


\subsection{Honeycomb lattice}

The classical honeycomb lattice is considered for the purpose of comparison. The sixfold symmetry provides transverse isotropy. Geometry can be described using the same parameters as for the rotachiral lattice. For a regular hexagonal honeycomb cell, $r$ and $D$ are not independent and $\delta=\sqrt{3}$ (cf. Fig. 4a). Also, $\beta=0.15$ and $\gamma \rightarrow+\infty$, which corresponds to $15 \%$ of volume fraction as for the other microstructures considered in this work. Unit-cell for this microstructure has been chosen hexagonal but it could have been square or rhomboid shaped as for the previous lattices (cf. Fig. 4b).

\section{Effective elastic properties}

Elastic moduli tensor $\underset{\approx}{C}$ is computed over a periodic unit-cell for each microstructure using Z-Set FE

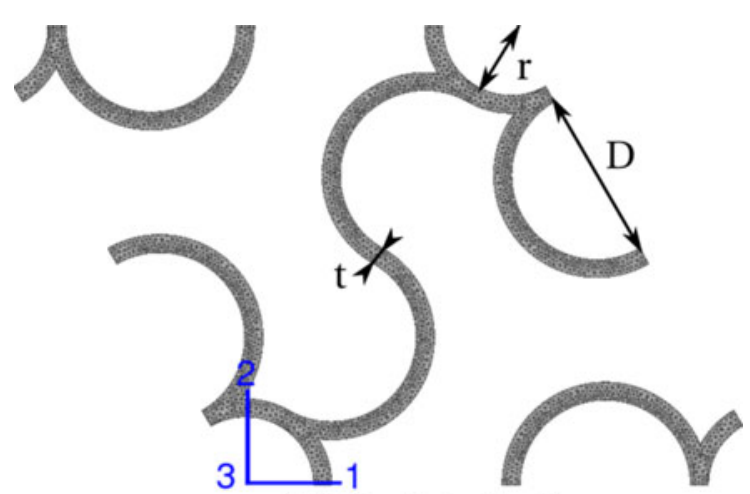

(a) Rotachiral unit-cell

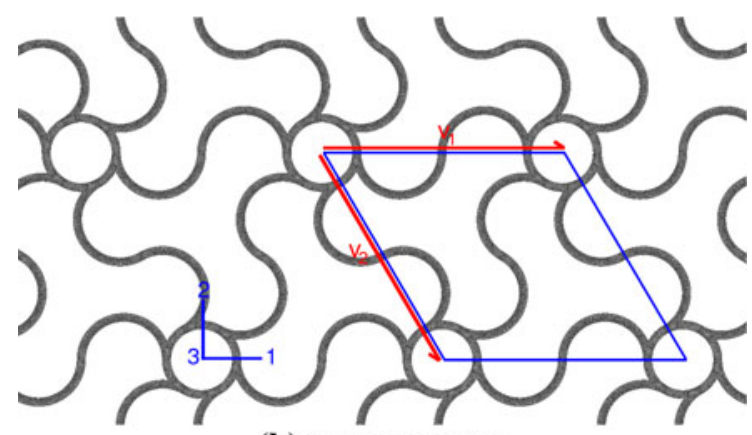

(b) Rotachiral lattice

Fig. 3 a Periodic cell with geometric parameters. b Rotachiral lattice with unit-cell (blue) and periodicity vectors $\underline{v_{1}}$ and $\underline{v_{2}}$ (red). (Color figure online)

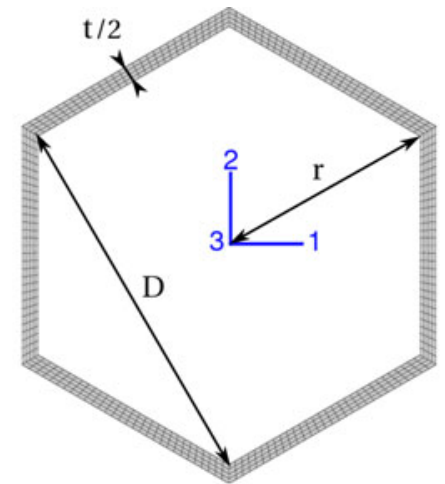

(a) Honeycomb unit-cell

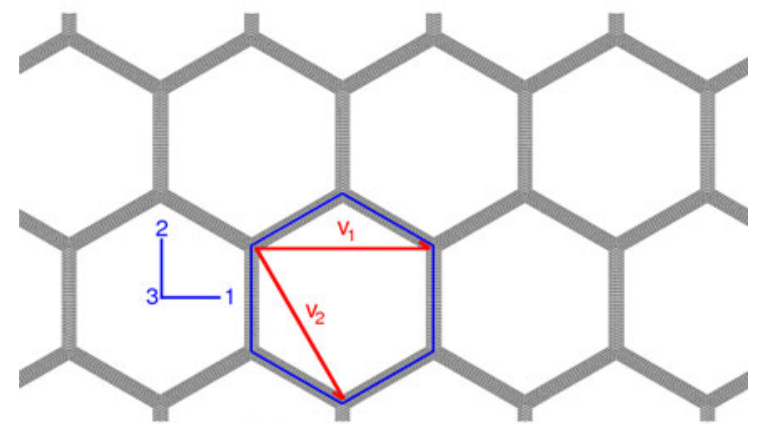

(b) Honeycomb lattice

Fig. 4 a Periodic cell with geometric parameters. b Honeycomb lattice with unit-cell (blue) and periodicity vectors $v_{1}$ and $\underline{v_{2}}(\mathrm{red})$

software ${ }^{1}$. Meshes are composed of volumic fullyintegrated quadratic elements, such as 10-node tetrahedra and 20-node hexahedra, taking into account the finite thickness of the microstructure components. Using the Euler-Bunge (1982) angles $\phi, \theta$ and $\psi$ as shown on Fig. 5, let us define three orthogonal vectors $\underline{l}, \underline{m}$ and $\underline{n}$ such as:

$$
\begin{aligned}
& {[\underline{l}]=\left[\begin{array}{c}
\cos (\phi) \cos (\psi)-\sin (\phi) \sin (\psi) \cos (\theta) \\
\sin (\phi) \cos (\psi)+\cos (\phi) \sin (\psi) \cos (\theta) \\
\sin (\psi) \sin (\theta)
\end{array}\right]} \\
& {[\underline{m}]=\left[\begin{array}{c}
-\cos (\phi) \sin (\psi)-\sin (\phi) \cos (\psi) \cos (\theta) \\
-\sin (\phi) \sin (\psi)+\cos (\phi) \cos (\psi) \cos (\theta) \\
\cos (\psi) \sin (\theta)
\end{array}\right]} \\
& {[\underline{n}]=\left[\begin{array}{c}
\sin (\phi) \sin (\theta) \\
-\cos (\phi) \sin (\theta) \\
\cos (\theta)
\end{array}\right]}
\end{aligned}
$$

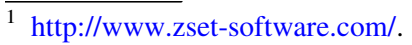




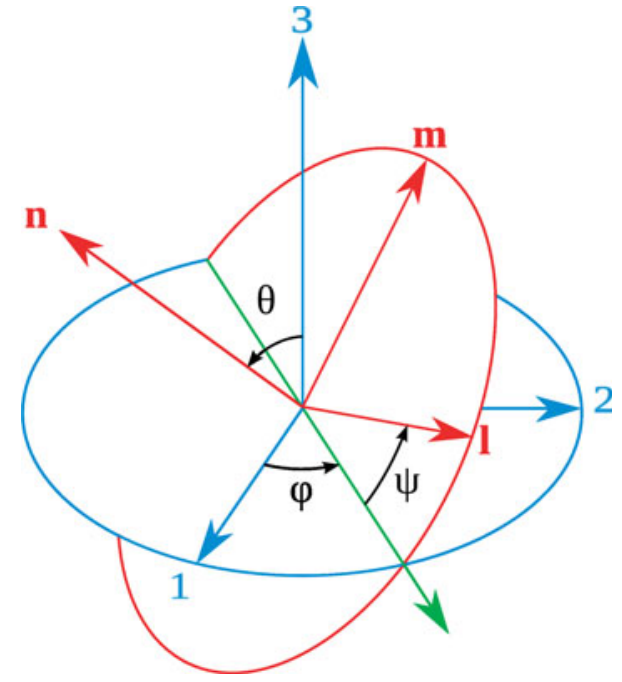

Fig. 5 Euler-Bunge angles

Using macroscopic strain and stress tensors $\underset{\sim}{E}(\phi, \theta, \psi)$ and $\sum_{\sim}(\phi, \theta, \psi)$, one can now define the Young's modulus $E(\underline{l})$ and effective Poisson's ratio $v^{*}(\underline{l}, \underline{m})$ for tension along direction $\underline{l}$ :

$E=\frac{\underline{l} \cdot \underset{\sim}{\Sigma} \cdot \underline{l}}{\underline{l} \cdot \underset{\sim}{E} \cdot \underline{l}}$

$v^{*}=-\frac{\underline{m} \cdot \underset{\sim}{E} \cdot \underline{m}}{\underline{l} \cdot \underset{\sim}{E} \cdot \underline{l}}$

If we now consider simple shear in the plane $(\underline{l}, \underline{m})$, the shear modulus $\mu(\underline{l}, \underline{m})$ can be defined as follows:

$2 \mu=\frac{\underline{l} \cdot \underset{\sim}{\Sigma} \cdot \underline{m}}{\underline{\underline{l}} \cdot \underset{\sim}{E} \cdot \underline{m}}$

For $\theta=\psi=0$, elastic moduli and Poisson's ratio are obtained in the plane $(1,2)$ of the microstructure as functions of $\phi$, we will refer to those as in-plane elastic properties. On the other hand, when $\phi=0$ and $\theta=\frac{\pi}{2}$, one obtains moduli and Poisson's ratio within plane $(1,3)$ as functions of $\psi$. These values will be considered as out-of-plane elastic properties.
For comparison purposes, normalized elastic moduli are defined using $f_{V}$, volume fraction of material, local constitutive isotropic elastic material parameters such as $E_{0}$ (Young's modulus) and $\mu_{0}$. Shear modulus $\mu_{0}$ is defined from $E_{0}$ and Poisson's ratio $v_{0}$ as follows:

$\mu_{0}=\frac{E_{0}}{2\left(1+v_{0}\right)}$

Thus, normalized Young's modulus $E^{*}$ is obtained as follows:

$E^{*}=\frac{1}{E_{0} f_{V}} E$

Normalized shear modulus $\mu^{*}$ is defined in this way:

$\mu^{*}=\frac{1}{\mu_{0} f_{V}} \mu$

In-plane elastic properties are shown in Table 1 and plotted against $\phi$ for the anti-tetrachiral cell on Figs. 6 and 7 (polar plots). The use of auxetic lattices in engineering applications might involve out-of-plane loading. Hence, $v^{*}, E^{*}$ and $\mu^{*}$ were also plotted against $\psi$ on Figs. 8, 9, 10, 11, 12, 13, 14 and 15. For this work, $E_{0}=210000 \mathrm{MPa}$ and $v_{0}=0.3$ are the isotropic elastic properties of the base material. Resulting elastic moduli tensors are presented hereafter as Eqs. 18-21. Components are expressed in MPa.

\subsection{Hexachiral lattice}

Elastic moduli tensor was computed for the hexachiral lattice as shown in Eq. 18. Transverse isotropy is verified since $\frac{C_{11}-C_{12}}{2}=C_{66}$.

$$
\underset{\approx}{[C}]=\left[\begin{array}{cccccc}
1650 & -1218 & 130 & 0 & 0 & 0 \\
-1218 & 1650 & 130 & 0 & 0 & 0 \\
130 & 130 & 31968 & 0 & 0 & 0 \\
0 & 0 & 0 & 5075 & 0 & 0 \\
0 & 0 & 0 & 0 & 5075 & 0 \\
0 & 0 & 0 & 0 & 0 & 1434
\end{array}\right]
$$

Table 1 In-plane Poisson's ratio and normalized elastic moduli

\begin{tabular}{lllll}
\hline & Hexachiral & Anti-tetrachiral & Rotachiral & Honeycomb \\
\hline$v^{*}$ & -0.74 & {$[-0.92 ; 0.69]$} & -0.26 & 0.92 \\
$E^{*}$ & $2.3 \times 10^{-2}$ & {$\left[4.3 \times 10^{-3} ; 2.7 \times 10^{-2}\right]$} & $2.7 \times 10^{-3}$ & $4.2 \times 10^{-2}$ \\
$\mu^{*}$ & $2.3 \times 10^{-1}$ & {$\left[6.6 \times 10^{-3} ; 8.8 \times 10^{-1}\right]$} & $9.6 \times 10^{-3}$ & $5.6 \times 10^{-2}$ \\
\hline
\end{tabular}




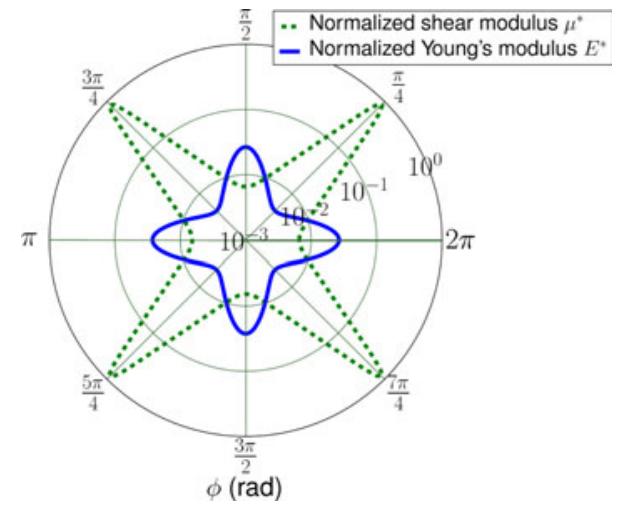

Fig. 6 Anti-tetrachiral lattice $(\theta=0, \psi=0)$

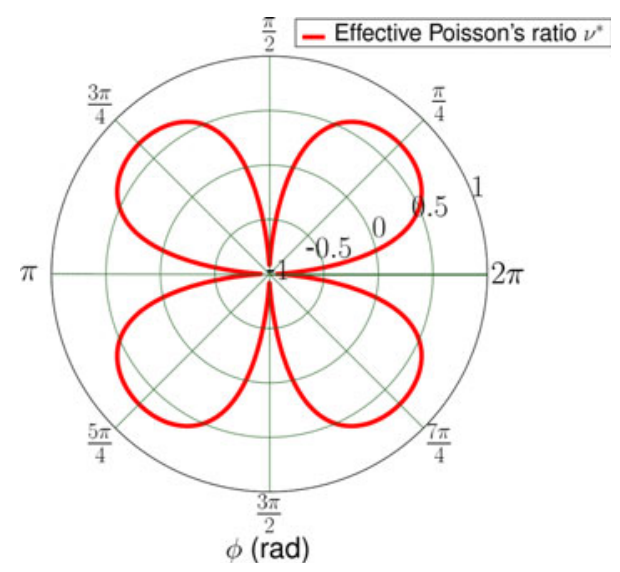

Fig. 7 Anti-tetrachiral lattice $(\theta=0, \psi=0)$

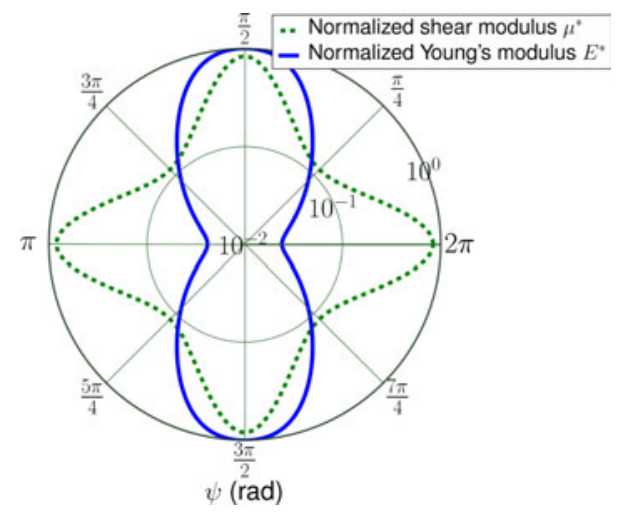

Fig. 8 Hexachiral lattice $\left(\theta=\frac{\pi}{2}, \phi=0\right)$

Components were used to obtain the in-plane properties gathered in Table 1. $v^{*}$ is underestimated compared to the value from Alderson et al. (2010), while our estimation of the normalized Young's modulus $E^{*}$

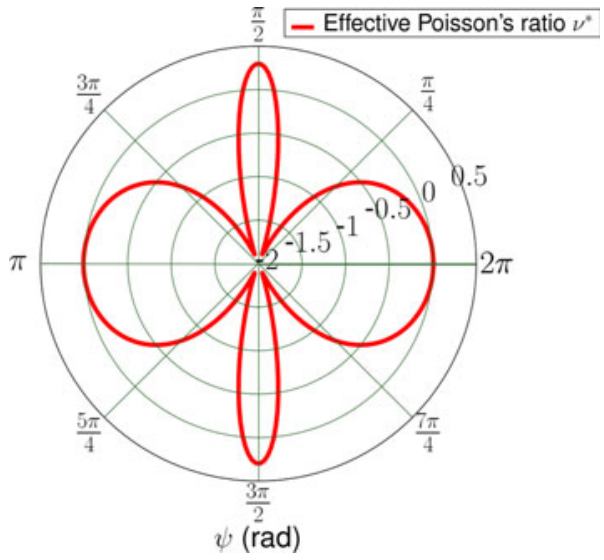

Fig. 9 Hexachiral lattice $\left(\theta=\frac{\pi}{2}, \phi=0\right)$

is higher. This is discussed later. Figure 8 shows an increase of $E^{*}$ when the material is streched out-of-plane, while reaching its maximum value along direction 3 $\left(\psi=\frac{\pi}{2}\right.$ or $\left.\psi=\frac{3 \pi}{2}\right)$. Figure 9 shows that Poisson's ratio $v^{*}$ is always negative, except for $\psi=0$ or $\psi=\pi$ where $v^{*}$ is close to 0 , and $\psi=\frac{\pi}{2}$ or $\psi=\frac{3 \pi}{2}$ where it takes the constitutive material value 0.3 . Normalized shear modulus $\mu^{*}$ fluctuates within a decade around the in-plane value depending on angle $\psi$.

\subsection{Anti-tetrachiral lattice}

While the small geometrical dissymmetry between direction 1 and 2 is theoretically responsible for orthotropic elasticity, it is negligible at the scale of the homogenized material since the obtained elastic moduli tensor (cf. Eq. 19) is representative of quadratic elasticity (invariant by rotation of $\frac{\pi}{2}$ in plane).

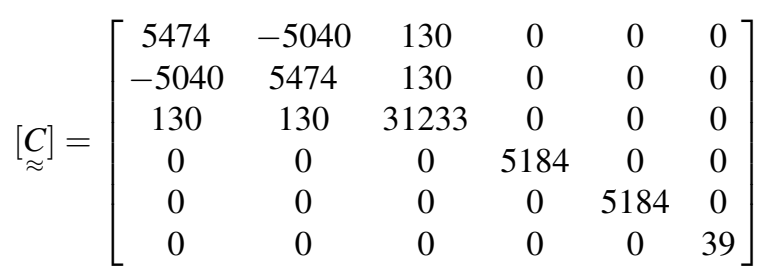

Figure 7 shows that in the cell's principal directions, $v^{*}$ is lower than the value from Alderson et al. (2010), for the same lattice with approximately the same geometric parameters, but the normalized Young's modulus $E^{*}$ is higher as shown on Fig. 6. Besides, $\mu^{*}$ fluctuates over 2 decades and reaches its minimum when $v^{*}$ is close to $-1 . v^{*}$ is negative for short angle 


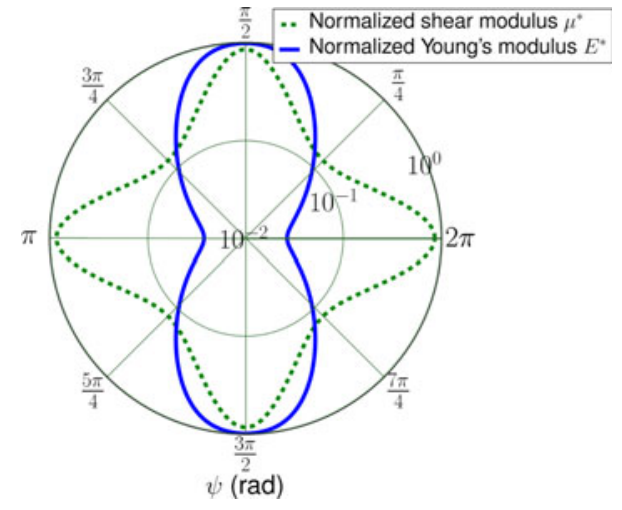

Fig. 10 Anti-tetrachiral lattice $\left(\theta=\frac{\pi}{2}, \phi=0\right)$

intervals around the principal directions of the cell. $E^{*}$ is varying over less than one order of magnitude depending on $\phi$. Normalized out-of-plane moduli are plotted on Fig. 10, which is very comparable with Fig. 8 in terms of values and angles. $E^{*}$ is higher than or equal to in-plane values. $v^{*}$ is always negative as shown on Fig. 11, except for $\psi=0$ or $\psi=\pi$ where $v^{*}$ is close to 0 , and $\psi=\frac{\pi}{2}$ or $\psi=\frac{3 \pi}{2}$ where it takes the bulk material value 0.3 as for the hexachiral lattice. $\mu^{*}$ fluctuates less with $\phi$ than with $\psi$.

\subsection{Rotachiral lattice}

The elastic moduli for the rotachiral lattice are given in Eq. 20. As for the hexachiral lattice, transverse isotropy is verified.

$$
\underset{\approx}{C}]=\left[\begin{array}{cccccc}
93 & -24 & 20 & 0 & 0 & 0 \\
-24 & 93 & 20 & 0 & 0 & 0 \\
20 & 20 & 31617 & 0 & 0 & 0 \\
0 & 0 & 0 & 3605 & 0 & 0 \\
0 & 0 & 0 & 0 & 3605 & 0 \\
0 & 0 & 0 & 0 & 0 & 59
\end{array}\right]
$$

The in-plane normalized moduli and effective Poisson's ratio are listed in Table 1. $E^{*}$ and $\mu^{*}$ are about one order of magnitude lower than for the hexachiral lattice. Figure 12 shows an increase of $E^{*}$ which fluctuates over 3 orders of magnitude when the material is streched outof-plane. $v^{*}$ is always negative as shown on Fig. 13, except for $\psi=0$ or $\psi=\pi$ where $v^{*}$ is close to 0 , and $\psi=\frac{\pi}{2}$ or $\psi=\frac{3 \pi}{2}$ where it reaches 0.3 , which is the constituent value. Normalized shear modulus $\mu^{*}$ is always higher than its in-plane counterpart.

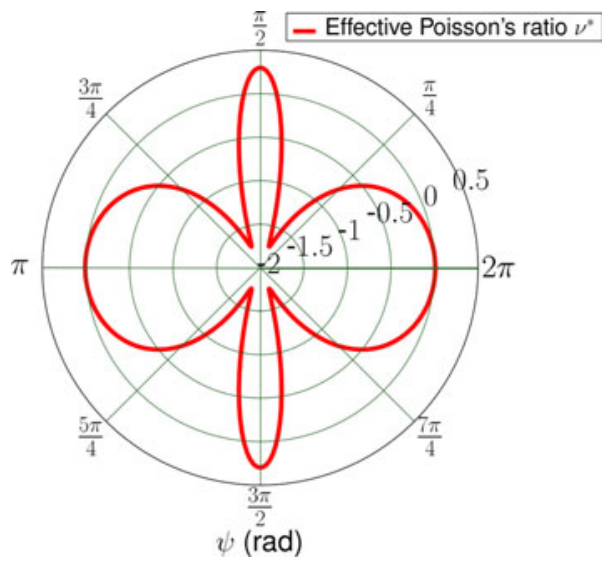

Fig. 11 Anti-tetrachiral lattice $\left(\theta=\frac{\pi}{2}, \phi=0\right)$

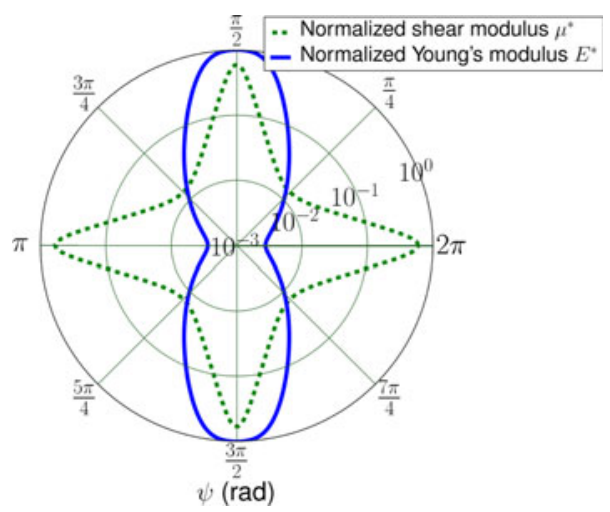

Fig. 12 Rotachiral lattice $\left(\theta=\frac{\pi}{2}, \phi=0\right)$

\subsection{Honeycomb lattice}

Elastic moduli tensor components were computed as shown in Eq. 21. Transverse isotropy is verified again.

$$
\underset{\approx}{\underset{\approx}{C}]}]=\left[\begin{array}{cccccc}
9945 & 9259 & 5761 & 0 & 0 & 0 \\
9259 & 9945 & 5761 & 0 & 0 & 0 \\
5761 & 5761 & 35070 & 0 & 0 & 0 \\
0 & 0 & 0 & 6512 & 0 & 0 \\
0 & 0 & 0 & 0 & 6512 & 0 \\
0 & 0 & 0 & 0 & 0 & 343
\end{array}\right]
$$

Components were used to obtain the in-plane properties gathered in Table 1. $v^{*}$ differs from the theoretical value of 1 , due to the beams slenderness hypothesis which is not fulfilled in our full-field simulations. Figure 14 shows an increase of $E^{*}$ when the material is streched out-of-plane. Surprisingly, out-of-plane, the effective Poisson's ratio $v^{*}$ shown on Fig. 15 is almost 


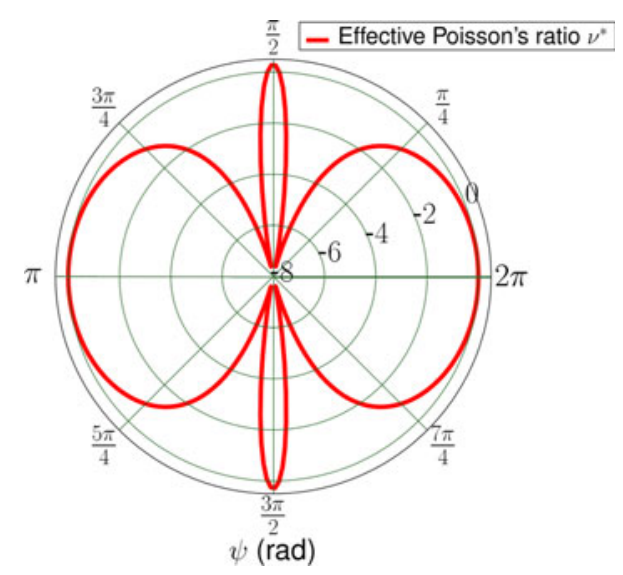

Fig. 13 Rotachiral lattice $\left(\theta=\frac{\pi}{2}, \phi=0\right)$

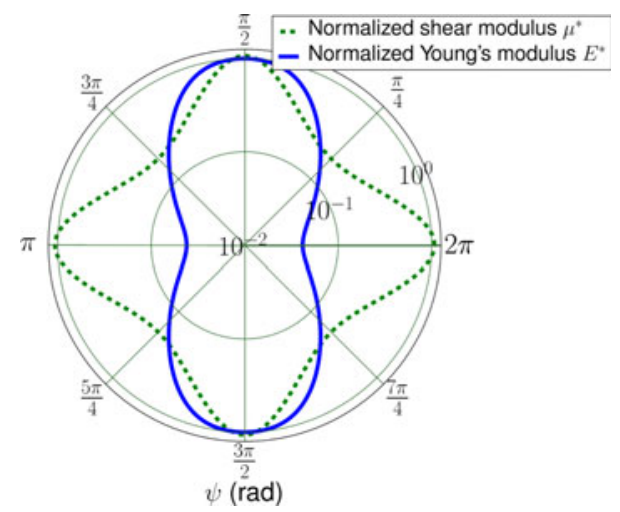

Fig. 14 Honeycomb lattice $\left(\theta=\frac{\pi}{2}, \phi=0\right)$

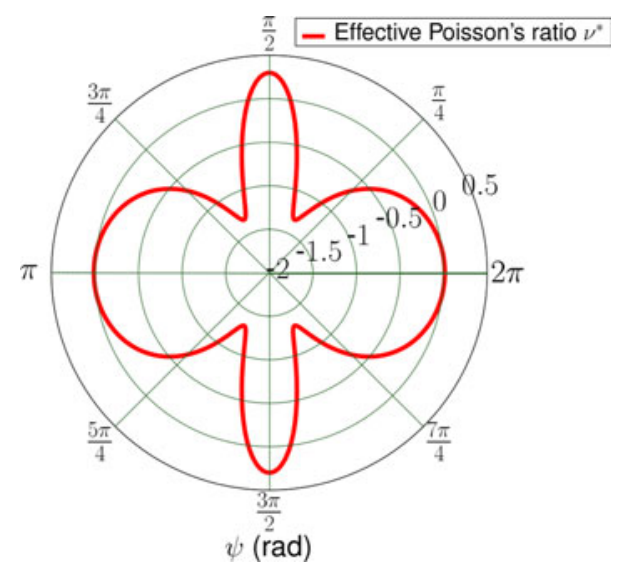

Fig. 15 Honeycomb lattice $\left(\theta=\frac{\pi}{2}, \phi=0\right)$

always negative for the honeycomb lattice, except for $\psi=0$ or $\psi=\pi$ where $v^{*}$ is close to 0 , and along direction $3\left(\psi=\frac{\pi}{2}\right.$ or $\left.\psi=\frac{3 \pi}{2}\right)$ where it takes the bulk material value 0.3 as for the other microstructures. Out-of-plane $\mu^{*}$ and $E^{*}$ are always equal or higher than their in-plane counterparts.

\subsection{Discussion}

Values obtained in this work for $E^{*}$ (cf. Table 1) exceed those from Alderson et al. (2010). This is due to the boundary conditions of the FEM problem. With periodicity over displacements and prescribed nodal forces, the configuration in Alderson et al. (2010) correponds to a static uniform boundary conditions micromechanical problem, which is known to lead to an underestimation of the elastic moduli (Kanit et al. 2003). On the other hand, the PBC problem gives exact results for an infinite medium. While the honeycomb cell exhibits the highest in-plane normalized Young's modulus, the hexachiral lattice presents a normalized shear modulus $\mu^{*}$ about four times higher. The hexachiral, anti-tetrachiral, rotachiral and honeycomb lattices all present a strong anisotropy when loaded out-of-plane (cf. Figs. 8, 10, 12, 14). Extreme Poisson's ratio value of -8 can be reached for the rotachiral lattice as shown on Fig. 13. It is worth noting that the anti-tetrachiral lattice presents a negative in-plane Poisson's ratio only for quite small angle intervals. Interestingly, for each microstructure, even the honeycomb lattice, $v^{*}$ is almost always negative when loaded out of plane (function of angle $\psi)$. The hexachiral, anti-tetrachiral and honeycomb lattices show comparable values in terms of magnitude for normalized elastic moduli as functions of $\psi$. For the same volume fraction, the impact on in and out-ofplane mechanical properties from the change in ligament geometry between hexachiral and rotachiral lattices is critical: circular ligaments give values which are more than one order of magnitude lower for both $E^{*}$ and $\mu^{*}$.

\section{3D auxetic microstructure: hexatruss lattice}

The hexatruss lattice is an extension of auxetic lattices to $3 \mathrm{D}$. It is very comparable to the unit-cell used in Doyoyo and $\mathrm{Hu}$ (2006) for modelling auxetic foams. The geometry of the cell is cubic, as shown on Fig. 16. The geometry can be described by lengths L, $1, t$ and angle $\omega$ as shown on Fig. 17. A new dimensionless parameter is defined in Eq. 22. L is not independent 


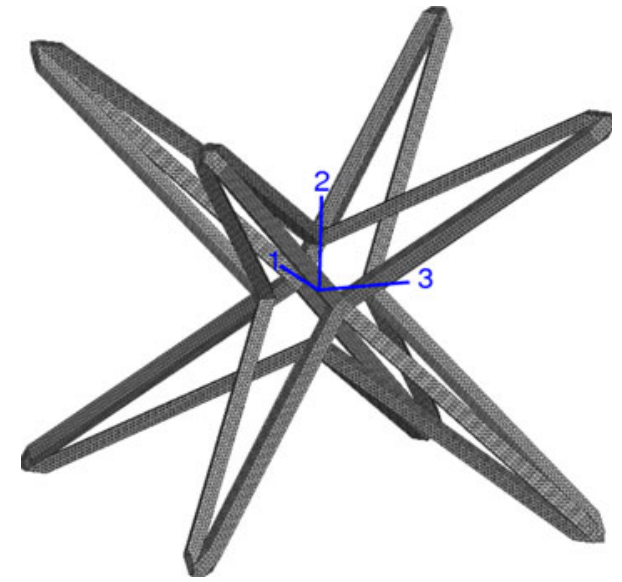

Fig. 16 Hexatruss unit-cell

from 1 and $\omega$, they are related to each other by the following equation: $\mathrm{L}=21 \cos ^{2} \omega$. For this work, $\omega=$ $\frac{\pi}{5}$ and $\zeta=15$, this corresponds to a volume fraction of $2.1 \%$.

$$
\zeta=\frac{1}{\mathrm{t}}
$$

The elastic moduli for the hexatruss lattice were computed in the same way as for 2D geometries. Cubic elasticity is verified, as shown here:

$[\underset{\approx}{C}]=\left[\begin{array}{cccccc}307 & 81 & 81 & 0 & 0 & 0 \\ 81 & 307 & 81 & 0 & 0 & 0 \\ 81 & 81 & 307 & 0 & 0 & 0 \\ 0 & 0 & 0 & 11342 & 0 & 0 \\ 0 & 0 & 0 & 0 & 11342 & 0 \\ 0 & 0 & 0 & 0 & 0 & 11342\end{array}\right]$

Components were used to plot elastic properties in Figs. 18 and 19. $v^{*}$ is negative for a large angle range, except near the principal directions of the cell, it reaches a minimal value of -0.97 for $\psi=\frac{\pi}{4}$. Figure 18 shows a strong increase of $\mu^{*}$ along the principal directions of the cell with a maximal normalized value of $12 . E^{*}$ is higher in diagonal directions than in the principal directions.

\section{Structural application of auxetics}

In order to conclude on the potential use of auxetic materials in engineering applications, we performed

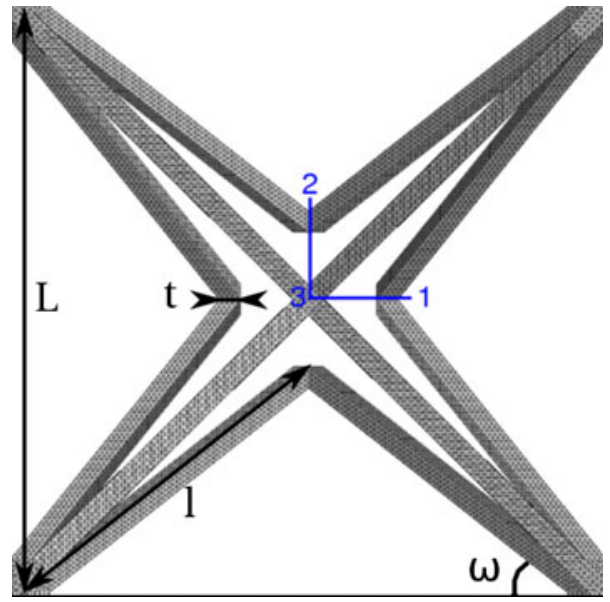

Fig. 17 Hexatruss periodic unit-cell with geometric parameters

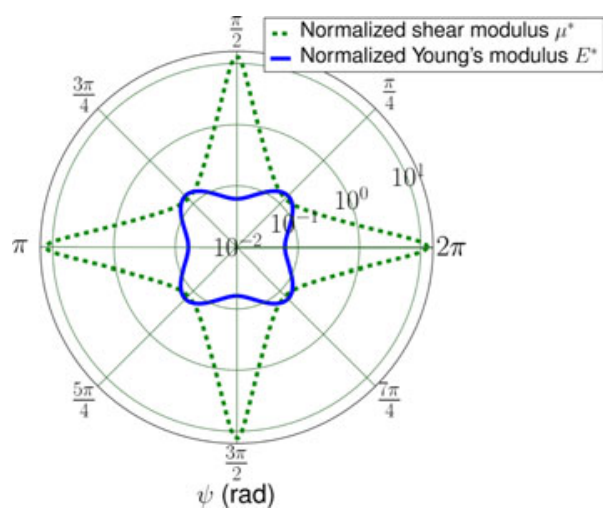

Fig. 18 Hexatruss lattice $\left(\theta=\frac{\pi}{2}, \phi=0\right)$

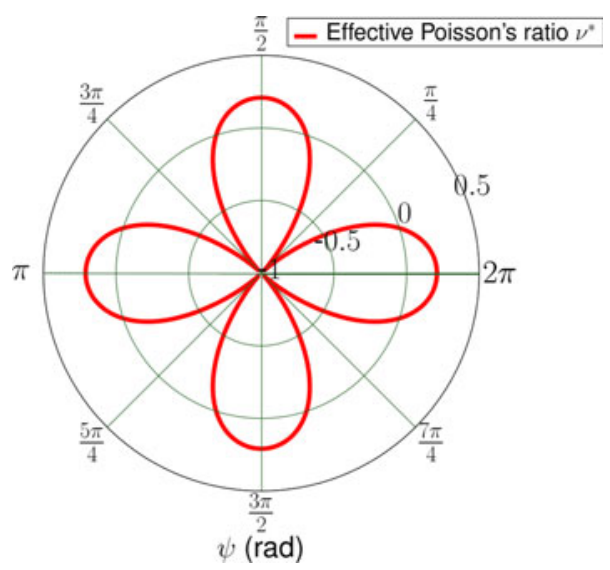

Fig. 19 Hexatruss lattice $\left(\theta=\frac{\pi}{2}, \phi=0\right)$ 


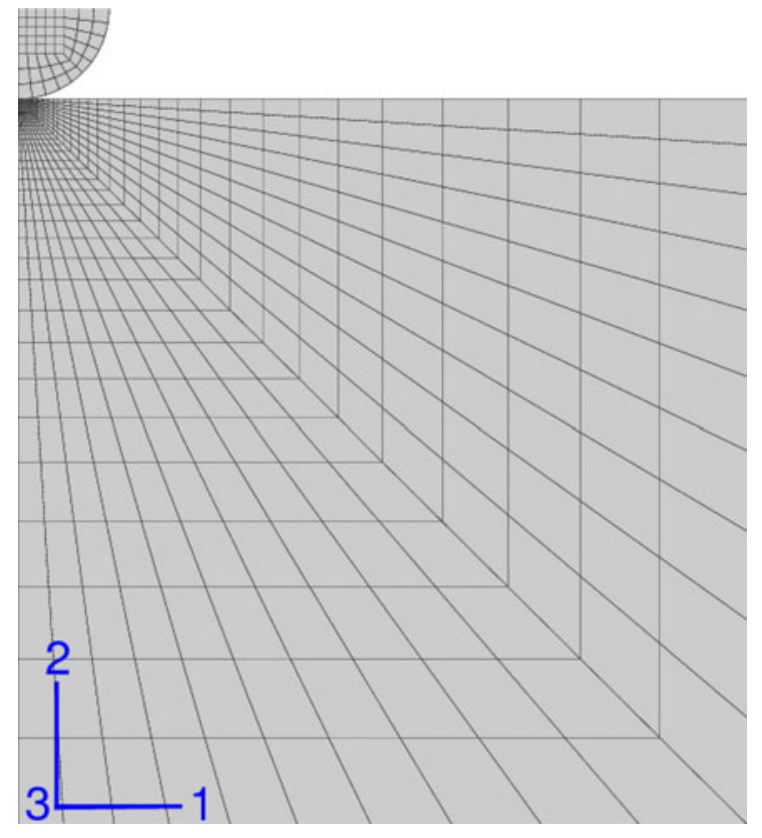

Fig. 20 FE mesh used for cylindrical indentation tests, here with orientation for in-plane indentation

simulations of both elastic cylindrical and spherical indentation on macroscopic homogenized models using elastic moduli determined in Sects. 4 and 5. Two sets of boundary conditions have been considered. For each set and each microstructure, indentation was performed along direction 2 with indented plane $(1,3)$ and direction 3 with indented plane $(1,2)$, respectively corresponding to in-plane and out-ofplane indentation tests. The material orientation used here is the microstructure orientation used in Figs. 1b, $2 \mathrm{~b}, 3 \mathrm{~b}, 4 \mathrm{~b}$ and 17 . Radius of the indentor $R=1 \mathrm{~mm}$ and maximum indentation depth $h_{s}=0.2 \mathrm{~mm}$.

\subsection{Cylindrical indentation}

First, an elastic cylindrical indentation test simulation was performed. The first loading case corresponds to the classical indentation test with prescribed displacement at the base of the indented medium along the direction of indentation. Symmetry conditions are also added in order to compute only half of the indentation problem. Loading is controlled by the displacement of the indentor. A 2D-mesh was used as shown on Fig. 20. Computations were done for both plane-strain and plane-stress assumptions. Force vs. indentation depth curves for both in-plane and out-of-plane

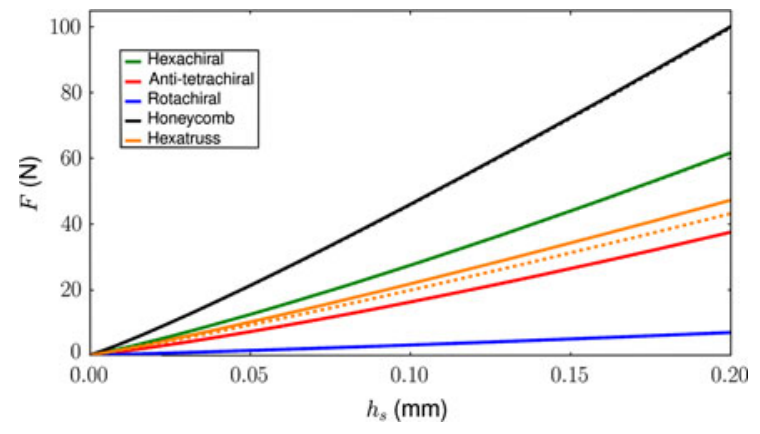

Fig. 21 Force versus indentation depth curves for cylindrical loading case 1 with indented plane $(1,3)$ —in-plane indentation

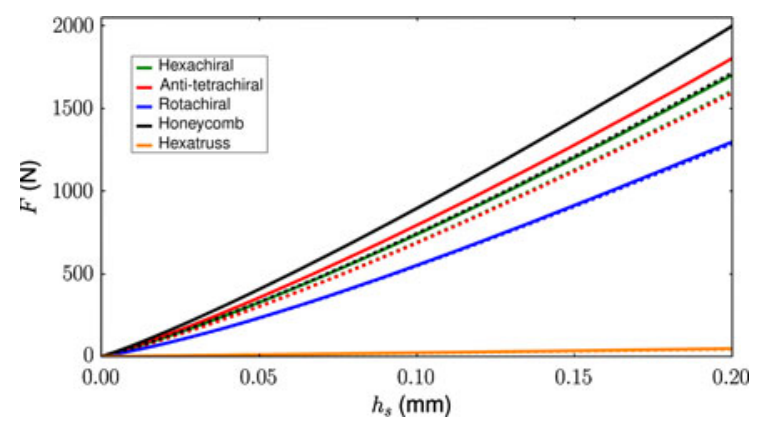

Fig. 22 Force versus indentation depth curves for cylindrical loading case 1 with indented plane $(1,2)$ —out-of-plane indentation

cylindrical indentations are presented in Figs. 21 and 22. Plain lines denote plane-strain assumption while dashed lines denote plane-stress. It is clear from Fig. 21 that the rotachiral lattice is not a good candidate for in-plane structural applications. On the other hand, results for the honeycomb cell are systematically good.

For the second loading case, the base of the indented medium is free, but displacement is now fixed to 0 at the external border of the indented solid. These boundary conditions are closer to those of an impact test, which relates more to what an hypothetical architectured sandwich panel would endure in use. For the second loading case, the hexachiral cell develops a higher strength in-plane than the honeycomb for $h_{s}=0.2 \mathrm{~mm}$ (cf. Fig. 23), this would advocate for the potential use of hexachiral cells for in-plane applications. Also, for the same loading case, the hexatruss lattice exhibits a very high in-plane strength for a volume fraction of $2.1 \%$ compared to the other cells with $15 \%$ volume fraction. The in- 


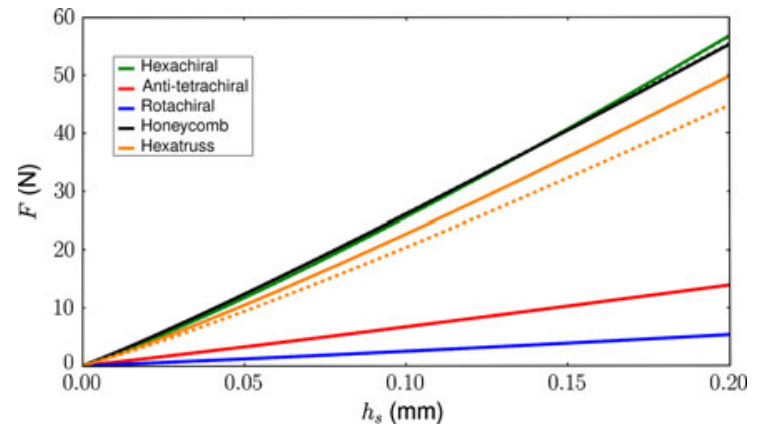

Fig. 23 Force versus indentation depth curves for cylindrical loading case 2 with indented plane (1,3) —in-plane indentation

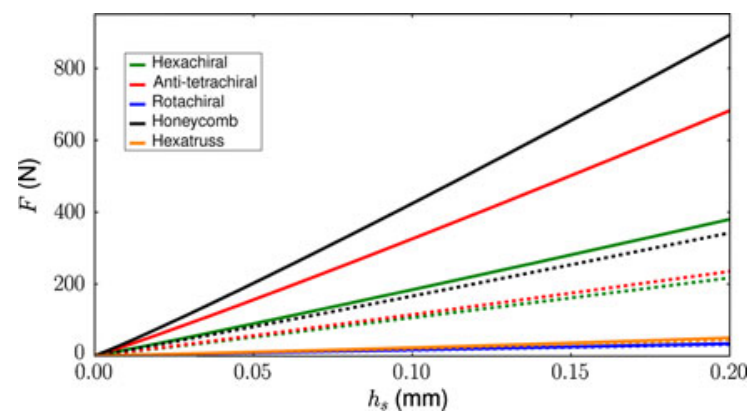

Fig. 24 Force versus indentation depth curves for cylindrical loading case 2 with indented plane $(1,2)$ - out-of-plane indentation

plane performance of the anti-tetrachiral cell is relatively low. If we now consider the out-of-plane performance of auxetics (cf. Figs. 22, 24), it is interesting to note the very low results of the hexatruss lattice compared to those from the extruded 2D geometries. This is due to its low volume fraction. The anti-tetrachiral cell exhibits high out-of-plane strength, comparable with the honeycomb strength level, for both loading cases. There are very strong discrepancies for the out-of-plane response of the rotachiral cell between loading case 1 and 2 .

\subsection{Spherical indentation}

In order to emphasize the structural applicability of auxetics, we performed spherical indentation simulation. Unlike the cylindrical indentation, we considered tridimensional meshes for the spherical indentation, as shown on Fig. 25. In order to reduce computation time, symmetry conditions were prescribed at face

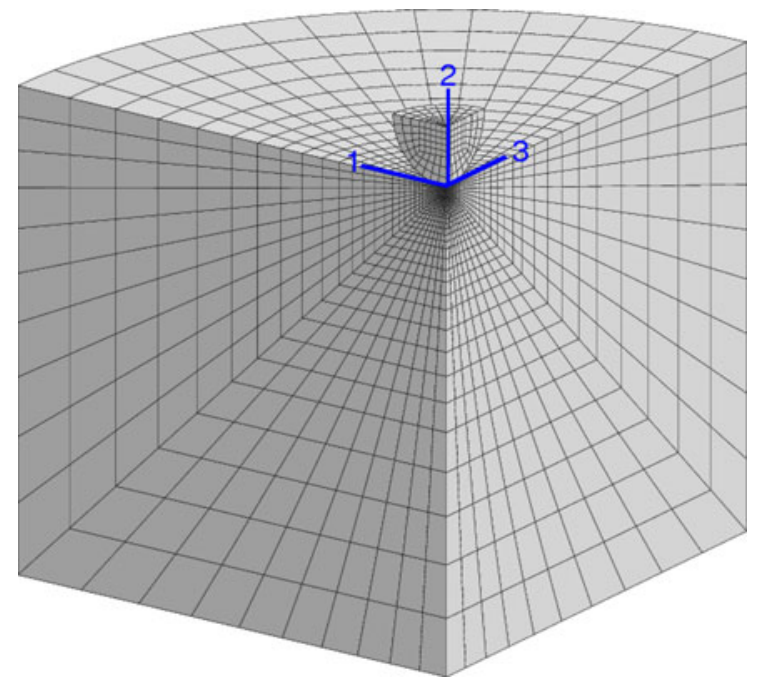

Fig. 25 Cross sectional view of FE mesh used for spherical indentation tests, here with orientation for in-plane indentation

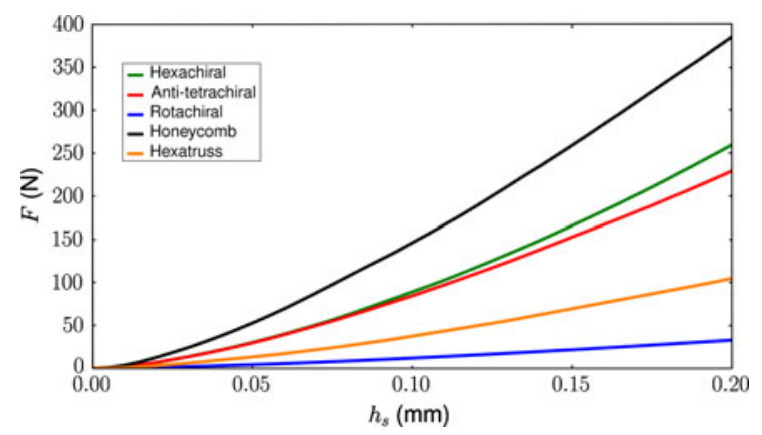

Fig. 26 Force versus indentation depth curves for spherical loading case 1 with indented plane $(1,3)$ - in-plane indentation

boundaries so that the FE problem is equivalent to a full spherical indentation simulation. Also, for the sake of simplicity, 8-node hexahedral and 6-node tetrahedral linear elements were chosen for this computation.

The first loading case corresponds to the same conditions as for the cylindrical indentation but with additional symmetry conditions in the planes transverse to the direction of indentation due to tridimensionality. Force versus indentation depth curves are shown on Fig. 26 for in-plane indentation and Fig. 27 for out-of-plane indentation. For both in-plane and out-of-plane indentations, the honeycomb cell exhibits the highest strength. Both hexachiral and antitetrachiral lattices develop a slightly lower but reasonnable strength. On the other hand, the rotachiral 


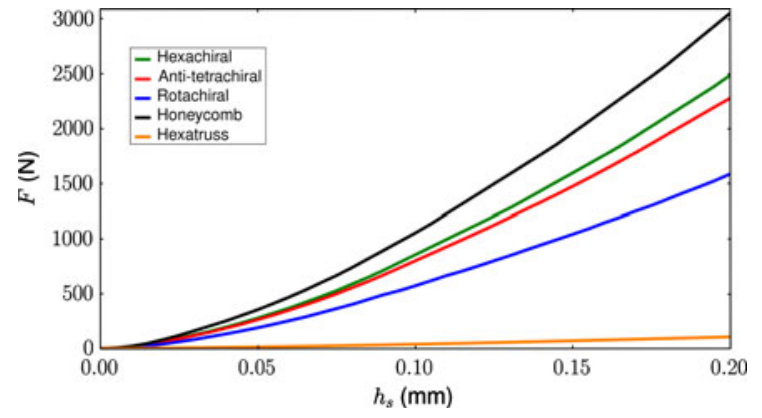

Fig. 27 Force versus indentation depth curves for spherical loading case 1 with indented plane $(1,2)$ —out-of-plane indentation

lattice does not seem adequate for such applications. The hexatruss cell performs quite well in-plane but exhibits poor properties out-of-plane as compared to the other cells, although the difference in volume fraction has to be taken into account.

For the second loading case, conditions are similar to those from the cylindrical indentation simulation but with additional symmetry conditions as for the spherical indentation above. Force versus indentation depth curves are shown on Fig. 28 for in-plane indentation and Fig. 29 for out-of-plane indentation. Once again, the honeycomb cell exhibits the highest strength for both orientations, and the hexachiral and anti-tetrachiral lattices are still competitive, at least in its principal directions for the latter. The rotachiral lattice is definitely inadequate as far as elastic energy is concerned. For in-plane structural applications, the hexatruss cell is a very competitive alternative to the honeycomb cell if volume fraction is considered.

\section{Conclusions and prospects}

Elastic moduli for three periodic auxetic 2D lattices, a $15 \%$ volume fraction honeycomb cell and a new 3D auxetic microstructure, have been computed using periodic homogenization technique coupled with FE. Anisotropy of in-plane and out-of-plane normalized elastic parameters was investigated. Each microstructure studied in this work was found to exhibit very strong anisotropy when loaded out-of-plane. Also, outof-plane effective Poisson's ratio is almost always negative for all the microstructures considered, even the classical honeycomb cell. With its circular (or elliptic) ligaments, the rotachiral lattice provides an

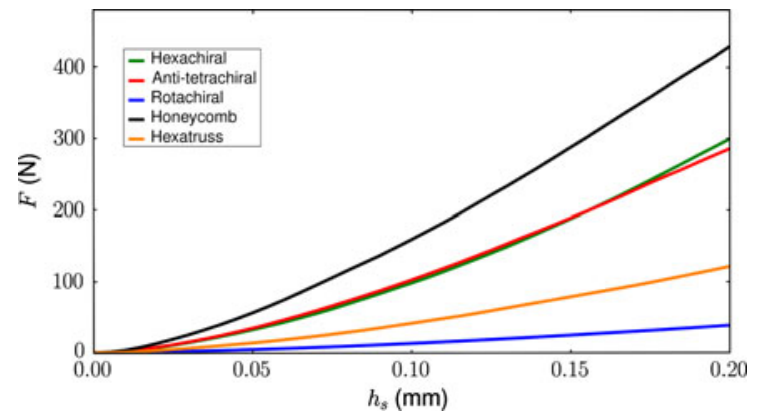

Fig. 28 Force versus indentation depth curves for spherical loading case 2 with indented plane (1,3) - in-plane indentation

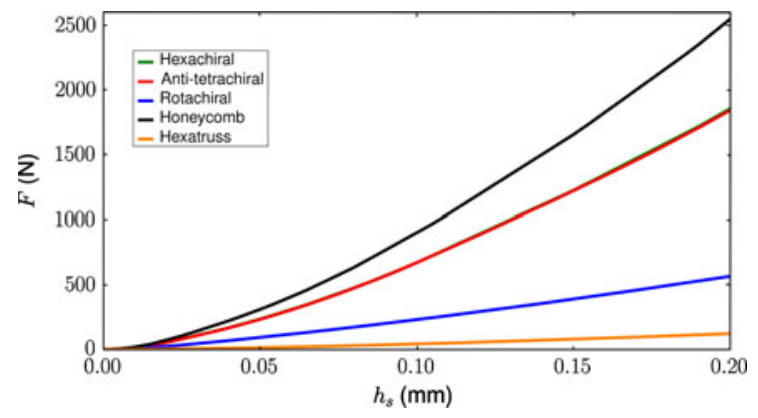

Fig. 29 Force versus indentation depth curves for spherical loading case 2 with indented plane $(1,2)$ —out-of-plane indentation

additional parameter for tuning the microstructure (Ashby and Bréchet 2003) for specific absorption properties. This lattice can exhibit highly negative Poisson's ratio when loaded out-of-plane. The orthotropy of the anti-tetrachiral lattice was investigated numerically, showing higher normalized Young's modulus $E^{*}$ in the principal directions of the cell, with in-plane auxetic effects restricted to short angle intervals around these directions. Such lattices could be used in replacement of traditional honeycomb-core for sandwich panels, especially if produced by extrusion. Results from structural computations were presented, advocating for further developments in the field of structural auxetics. While the honeycomb cell exhibits a constantly high indentation strength, auxetic lattices can be competitive if shear is involved, especially the hexachiral and anti-tetrachiral lattices. The hexatruss 3D lattice gave promising results in terms of indentation strength, especially if volume fraction or density is the key parameter for designing a structure. It would be interesting to compare the hexatruss mechanical performance with those of other 
tridimensional strut-lattices. Nevertheless, due to the importance of sandwich skins in the strength of composite structures, those will be taken into account and their influence investigated in subsequent works. While not mentioned in this work, auxetic samples are being made using selective laser melting, a powder metallurgy process, extending one's microstructural design spectrum from 2D to 3D. This method has been used successfully by others for auxetics (Huang and Blackburn 2002; Schwerdtfeger et al. 2010; Mitschke et al. 2011). New tridimensional auxetic microstructures are currently being developed using this technique coupled with numerical homogenization. Numerical results will be confronted to experimental data currently being obtained. The influence of auxeticity on plasticity has already been partly investigated (Dirrenberger et al. 2012) but further studies have to be conducted in this direction. For industrial applications, non-linear phenomena such as buckling will have to be taken into account.

Acknowledgments This work is part of the MANSART (Architectured sandwich materials) project ANR-08-MAPR0026. Financial support of ANR is gratefully acknowledged.

\section{References}

Alderson, K.L., Pickles, A.P., Neale, P.J., Evans, K.E.: Auxetic polyethylene - the effect of a negative Poisson ratio on hardness.Acta Metall. Mater. 42(7), 2261 (1994)

Alderson, K.L., Fitzgerald, A., Evans, K.E.: The strain dependent indentation resilience of auxetic microporous polyethylene. J. Mater. Sci. 35(16), 4039 (2000)

Alderson, v., Alderson, K.L., Attard, D., Evans, K.E., Gatt, R., Grima, J.N., Miller, W., Ravirala, N., Smith, C.W., Zied, K.: Elastic constants of 3-, 4-and 6-connected chiral and anti-chiral honeycombs subject to uniaxial in-plane loading. Compos. Sci. Technol. 70(7), 1042 (2010)

Almgren, R.F.: An isotropic three-dimensional structure with poisson's ratio $=-1$. J. Elast. 15, 427 (1985)

Ashby, M.F., Bréchet, Y.: Designing hybrid materials. Acta Mater. 51, 5801 (2003)

Bunge, H.J.: Texture Analysis in Materials Science. Butterworths, London (1982)

Caddock, B.D., Evans, K.E.: Microporous materials with negative poisson's ratios: I. Microstructure and mechanical properties. J. Phys. D 22, 1877 (1989)

Chen, C.P., Lakes, R.S.: Micromechanical analysis of dynamic behavior of conventional and negative Poisson's ratio foams. J. Eng. Mater. Technol. 118(3), 285 (1996)

Choi, J.B., Lakes, R.S.: International Journal of Fracture 80, 73 (1996)
Dirrenberger, J., Forest, S., Jeulin, D., Colin, C.: Homogenization of periodic auxetic materials. Procedia Eng. 10, 1847 (2011). doi:10.1016/j.proeng.2011.04.307

Dirrenberger, J., Forest, S., Jeulin, D.: Elastoplasticity of auxetic materials. Comput. Mater. Sci. (2012). doi:10.1016/ j.commatsci.2012.03.036

Doyoyo, M., Hu, J.W.: Plastic failure analysis of an auxetic foam or inverted strut lattice under longitudinal and shear loads. J. Mech. Phys. Solids 54, 1479 (2006)

Evans, K.E., Nkansah, M.A., Hutchinson, I.J., Rogers, S.C.: Molecular network design. Nature 353, 124 (1991a)

Evans, K.E.: The design of doubly curved sandwich panels with honeycomb cores. Compos. Struct. 17(2), 95 (1991b)

Gaspar, N., Ren, X.J., Smith, C.W., Grima, J.N., Evans, K.E.: Novel honeycombs with auxetic behaviour. Acta Mater. 53, 2439 (2005)

Huang, X., Blackburn, S.: Developing a new processing route to manufacture honeycomb ceramics with negative Poisson's ratio. Key Eng. Mater. 206-213, 201 (2002)

Jean, A., Engelmayr, G.C.: Finite element analysis of an accordion-like honeycomb scaffold for cardiac tissue engineering. J. Biomech. 43, 3035 (2010)

Kanit, T., Forest, S., Galliet, I., Mounoury, V., Jeulin, D.: Determination of the size of the representative volume element for random composites: statistical and numerical approach. Int. J. Solids Struct. 40, 3647 (2003)

Lakes, R.S.: Foam structures with a negative poisson's ratio. Science 235, 1038 (1987)

Lakes, R.S.: Deformation mechanisms in negative poisson's ratio materials: structural aspects. J. Mater. Sci. 26, 2287 (1991)

Milton, G.W.: Composite materials with poisson's ratios close to -1 . J. Mech. Phys. Solids 40(5), 1105 (1992)

Lakes, R.S.: Materials with structural hierarchy. Nature 361, $511(1993)$

Lipsett, A.W., Beltzer, A.I.: Reexamination of dynamic problems of elasticity for negative Poisson's ratio. J. Acoust. Soc. Am. 84(6), 2179 (1988)

Madi, K., Forest, S., Cordier, P., Boussuge, M.: Numerical study of creep in two-phase aggregates with a large rheology contrast: implications for the lower mantle. Earth Planet. Sci. Lett. 237(1-2), 223 (2005). doi:10.1016/j.eps1.2005.06.027

Mitschke, H., Schwerdtfeger, J., Schury, F., Stingl, M., Körner, C., Singer, R.F., Robins, V., Mecke, K., Schröder-Turk, G.E.: Finding auxetic frameworks in periodic tessellations. Adv. Mater. 23, 2669 (2011)

Prall, D., Lakes, R.S.: Properties of a chiral honeycomb with a poisson's ratio of -1 . Int. J. Mech. Sci. 39(3), 305 (1997)

Scarpa, F., Yates, J.R., Ciffo, L.G., Patsias S.: Dynamic crushing of auxetic open-cell polyurethane foam. J. Mech. Eng. Sci. 216(12), 1153-1156 (2002)

Schwerdtfeger, J., Heinl, P., Singer, R.F., Körner, C.: Auxetic cellular structures through selective electron-beam melting. Phys. Status Solidi 247(2), 269 (2010)

Spadoni, A.: Application of chiral cellular materials for the design of innovative components. Ph.D. thesis, Georgia Institute of Technology. (2008)

Spadoni, A., Ruzzene, M., Gonella, S., Scarpa, F.: Phononic properties of hexagonal chiral lattices. Wave Motion 46(7), 435 (2009) 